\title{
ReFresh: Retaining First Year Engineering Students and Retraining for Success
}

\author{
Neil Shyminsky and Lesley Mak \\ University of Toronto \\ lmak@ecf.utoronto.ca
}

\begin{abstract}
Student retention and support are key priorities at the University of Toronto Faculty of Applied Science and Engineering, as is evidenced by a first year to second year average retention rate of $91 \%$ over the past 4 years. At $U$ of $T$ Engineering, academic standing and registration are determined on a term-by-term basis. As a result, student performance in the fall term can result in obligatory withdrawal from their studies in January, including first year students. While approximately only $4 \%$ of the first year class have to withdraw, the impact can be very distressing for the individual student, as his/her plans for the year are disrupted and their confidence shaken. Withdrawal from studies can occur for many reasons such as insufficient academic background from their high school, personal crisis, low resilience and lack of study skills and these struggles can be especially acute for international students. The ReFresh Program gives these students an opportunity to continue their education while learning from their mistakes. A small classroom environment allows students to integrate into a collaborative community, create study groups with classmates, communicate easily with their instructors and TA's and benefit from constant support from First Year Office staff. This format helps students deal with the personal and academic challenges that prevented them from performing at their best in the fall term, relearn the foundations and key concepts of Calculus, Physics, Computer Programming, Chemistry and Linear Algebra and develop a plan to be successful for the next fall to repeat their first semester. This paper will discuss the structure of the ReFresh program as well as the impact on student success, student development and retention.
\end{abstract}

Keywords: Retention, first year experience, academic skills, student success, student development

\section{INTRODUCTION}

Student transition into first year of university is a welldocumented challenge that institutions across the world try to support. Engineering has a reputation for being especially difficult as the foundations of math, physics, computers, chemistry, and electricity often have to be established in their first year courses. This can lead to more class hours and assignments than students are accustomed to handling, and when standard transition challenges like living away from home or long commutes as well as new found independence and unfamiliar environments are added to the mix, many students can fail to perform academically. Failing a course, or worse, failing an entire semester can have devastating impacts on a students' self-esteem and disrupt their academic plans. While student transition and attrition in the past was blamed on the student for a lack of intelligence or drive to succeed, a more modern view of academic success indicates a relationship between the student, his/her community of peers, the teachers and the support systems the institution provides to create a healthy living and learning environment. [1]

At the University of Toronto Faculty of Applied Science and Engineering, first year students can be withdrawn from their academics in January after their first fall semester due to the promotion regulations. This sudden departure from academics can be devastating it causes students to re-evaluate their reasons for being at $\mathrm{U}$ of $\mathrm{T}$ Engineering as well as their work ethic, study skills and their understanding of the material. Students who once were able to cram for final exams in high school and receive $>90 \%$ are now told they've failed for the first time and are no longer registered in classes. Students with 45$50 \%$ sessional averages are guaranteed to return the subsequent September to repeat first year but those below $45 \%$ have to submit an application and their case is reviewed to determine if they will be allowed to re-enrol to repeat first year. For international students, many of them have to return home as their visas won't allow them to stay in Toronto if they aren't enrolled in classes. This return to their community and family can cause a great deal of shame.

As a result, the First Year Office (FYO) at $\mathrm{U}$ of $\mathrm{T}$ Engineering created a program called ReFresh in the 2008-2009 school year that would allow students to stay enrolled in classes but through a program that would reteach them the academic foundations of first year engineering while building effective goal setting, study 
skills, time management and emotional intelligence. This following report gives an overview of the program and presents an analysis of various statistics derived from the 2009-2012 ReFresh programs in order to gauge its effectiveness.

\section{PROGRAM STRUCTURE}

Though the ReFresh program has changed and evolved slightly during the past five years, the FYO has kept the core structure consistent. Students are able to register for ReFresh in January after receiving their marks up until the program's start and the program runs for 10 weeks February-April with assignments, quizzes, midterms and final exams or projects. Students pay approximately $\$ 3500$ for the entire program and stay registered with the university as part time students, giving them full access to all of the services including residence, athletics, cocurriculars, health services and counseling.

Students have to attend the entire ReFresh set of courses and Physics, Calculus, Linear Algebra, Chemistry and Programming courses, each with 2 hours of lecture and 2 hours of tutorials per week comprise of the technical courses. Each week also includes 3 hours of general seminars and 2 hours of study hall per week. Graduate students (one instructor and one TA per course) are hired based on their understanding of student transition issues as well as their ability to teach foundational concepts simply and within the greater context of engineering. The courses begin their curriculum at a high school level of difficulty and speed but then progress to first year university level difficulty once students start to understand the core concepts. Instructors and TA's also take feedback from the students seriously and will slow down or review material if the class is struggling. The FYO team work closely with the Instructor and TA Teaching Team through weekly meetings to offer support for the challenges of teaching such a unique class of students who vary on the spectrum of engagement and skills. The teaching team also receive a report based on student feedback halfway through the program and again at the end so they learn how to improve their skills.

Aside from the technical material, students are challenged to reflect on their challenges and struggles through several methods. The weekly seminars are taught by staff in the FYO as well as faculty and staff and students learn life skills such as how to set effective goals, how to re-evaluate their time management approach, take useful notes in class and improve their communication skills. Students complete various kinds of marked assignments for their seminars such as time management templates to measure how they spend their time and essays on why they want to return to $U$ of $T$ Engineering. Students also have to write weekly journals to answer questions such as "What is your biggest flaw? How do you turn that flaw into a positive trait?" and "Describe a stressful situation you experienced. How did you cope?” Staff in the FYO split up the 15-30 students in the ReFresh class and read their students' journals and respond to them. Staff also have regular one-on-one meetings with the students in their journal group to talk further about their journals or discuss other challenges or successes they were encountering. One-on-one attention from the FYO staff ensured students were accessing appropriate services as needed such as accessibility services and counselling.

Students receive a "report card" halfway through the program as well as at the end. The end of term program gives the students a mark for each of the 5 technical courses as well as a mark for the seminars. They get a final ReFresh mark that is an average of all 6 marks and they must obtain $>70 \%$ to pass ReFresh. If they pass they receive an unweighted credit (CR) notation on their transcript, as opposed to no credit (NCR) for failing. Students are able to use their ReFresh marks if they want to apply to return to $\mathrm{U}$ of $\mathrm{T}$ Engineering or if they want to apply to a different faculty or institution. Students who pass ReFresh are usually granted re-admission to the Faculty to repeat first year due to their proven abilities and personal growth. Many students who complete the ReFresh program do return to $\mathrm{U}$ of $\mathrm{T}$ Engineering to repeat their first year as can be seen in the results below.

\section{EFFECTIVENESS OF REFRESH}

As the program has been running for 5 years, we were able to look back on the performance of ReFresh students to see how effective it is. In the first part of our research we examined all four years of ReFresh and used correlation scores to evaluate the relationship between a failed or withdrawn student's ReFresh grades and both their first and second attempts at the Fall term. Does a ReFresh student's performance in the program give some indication of how s/he will perform when they return to attempt the Fall of first-year again? Our conclusion, based on the data to this point, is that the $1^{\text {st }}$ attempt performance provides basically no value in predicting a $2^{\text {nd }}$ attempt, that ReFresh performance is a much better predictor of $2^{\text {nd }}$ attempt performance, and, lastly, that the top ReFresh performances (students who score above $80 \%$ in ReFresh) are particularly good indicators of subsequent success in a students' $2^{\text {nd }}$ attempt.

In the second part, we specifically compared two kinds of students who were repeating Fall of first-year: ReFresh and non-ReFresh returners. At first glance, there is little difference in their $2^{\text {nd }}$ Fall and their Winter results. However, the top performers in ReFresh outpace the average returning student by a significant margin, and in a comparison of the subset ReFresh and non-ReFresh 
students who finish their $1^{\text {st }}$ attempt with an average of 45$49 \%$, the ReFresh group is the clear winner in the Fall term, though the difference is very small in the Winter. As well, ReFresh proves to be very effective when it comes to student retention, resulting in a repeater-rate that is roughly double that seen in the non-ReFresh student population.

All this said, there are some serious issues surrounding selection bias that make it difficult to analyze the data in this second part. Most significantly, we explain why we suspect that ReFresh students are, on the whole, already weaker students than their non-ReFresh counterparts. Further analysis with supporting qualitative data interviews with past students, both ReFresh and nonReFresh, in a later review and paper would serve to contextualize the results and lend some needed perspective to this analysis.

\subsection{Correlation between ReFresh and $2^{\text {nd }}$ Attempt Performance}

We started our review of ReFresh with a single question: Does ReFresh actually change the trajectory of a student who has previously failed, or does it simply give an extra push to those who were already on the bubble, while giving false hope to those who had previously failed by a significant margin? For instance, was the student who scored $48 \%$ in their $1^{\text {st }}$ attempt at Fall term getting a $63 \%$ in the $2^{\text {nd }}$ attempt, while the student who scored $30 \%$ was merely improving to $45 \%$ ? If so, if the strong and weak students have the same relative standing and the $1 \mathrm{st}$ attempt is a good predictor of performance in the 2nd attempt, regardless of ReFresh performance, then it would be difficult to argue that ReFresh is having much of an impact. Conversely, does ReFresh serve as a better predictor of academic performance in the $2^{\text {nd }}$ attempt? If the strongest ReFresh students are also the strongest repeater students - or at least the strongest repeaters among ReFreshers - that would suggest that the program is teaching them something that they can carry over, that has value beyond ReFresh.

We examined the first four years of ReFresh using $\mathrm{R}$ scores (Table 1), which is a method of measuring the correlation between paired sets of numbers. To be perfectly clear: We're not trying to determine whether ReFresh actually "helps" or “improves" student performance. That question can't be answered by this kind of stats-based analysis. Rather, we're trying to see whether the relationships between performances in

1. $1^{\text {st }}$ and $2^{\text {nd }}$ attempt

2. ReFresh and $2^{\text {nd }}$ attempt

are relatively strong or weak, from which we can draw some speculative conclusions. To repeat the cliché, correlation does not necessarily imply causation, though it's certainly a possibility in this situation. But since we're talking about education and a program where one of its goals is to prepare students for a return to this Faculty, we'd like to see the ReFresh-2nd attempt comparison produce a positive correlation - an $r^{2}$ above 0 , the higher the better and a higher correlation than that between 1st attempt and 2nd attempt. If these conditions are met then we can reasonably intuit a causal relationship of some kind.

Table 1: ReFresh student performance

\begin{tabular}{|l|c|c|c|c|}
\hline $\begin{array}{l}\text { Student } \\
\text { population }\end{array}$ & $\begin{array}{l}\text { 1st \& } \\
\text { 2nd } \\
\text { attempt } \\
\left(\mathrm{r}^{2}\right)\end{array}$ & $\begin{array}{l}\text { RF \& } \\
\text { 2nd } \\
\text { attempt } \\
\left(\mathrm{r}^{2}\right)\end{array}$ & $\begin{array}{l}\text { RF avg } \\
(\%)\end{array}$ & $\begin{array}{l}\text { 2nd } \\
\text { Attempt } \\
\text { Fall avg } \\
(\%)\end{array}$ \\
\hline $\begin{array}{l}\text { All RF } \\
(\mathrm{n}=64)\end{array}$ & 0.01 & 0.18 & 72 & 61 \\
\hline $\begin{array}{l}>80 \% \text { in } \\
\text { RF (n=23) }\end{array}$ & 0.22 & 0.44 & 88 & 69 \\
\hline $\begin{array}{l}\text { Guaranteed } \\
\text { return } \\
\text { (n=31) }\end{array}$ & 0 & 0.25 & 70 & 66 \\
\hline $\begin{array}{l}\text { Must } \\
\text { reapply } \\
\text { (n=33) }\end{array}$ & -0.04 & 0.35 & 75 & 59 \\
\hline
\end{tabular}

Table 1 - Comparison of various populations of ReFresh (RF) students from 2008-2012. "Guaranteed return" were those with a first attempt average of $>45 \%$ and "Must reapply" was $<45 \%$

These are very small numbers of students, so it goes without saying that we're drawing some very tentative conclusions. That said, there are a few things worth pointing out. There is no correlation between all students' $1^{\text {st }}$ and $2^{\text {nd }}$ attempt at the Fall term. If we were asked, on the basis of a spreadsheet alone, to guess whether a student with a 25 or 45 average in their $1^{\text {st }}$ attempt would ultimately do better in their $2^{\text {nd }}$ attempt, it is effectively a toss-up. The 1st attempt has no predictive value for the 2nd attempt. By comparison, ReFresh has much more predictive value. Among the entire population of ReFreshers, the correlation is still weak; however, when we restrict analysis to those who do very well in ReFresh (have an $80 \%$ average or higher), a moderate correlation emerges. Predictably, these students do very well in their $2^{\text {nd }}$ attempt at the Fall term, scoring an average just under $70 \%$. What we can say pretty strongly, then, is that a strong performance in ReFresh is indeed predictive of modest future success.

\subsection{Comparison of RF and non-RF repeaters}


In this comparison, we looked at the data for students beginning in 2008, 2009, and 2010, and specifically examined two distinct kinds of students who were repeating Fall of first-year: ReFresh and non-ReFresh returners. (Table 2) The question we attempted to answer was whether ReFreshers performed similarly, worse, or better than their repeater peers who did not take ReFresh. Importantly, we looked not only at each student's $2^{\text {nd }}$ attempt at the Fall term, but also their $1^{\text {st }}$ attempt at the Winter term.

Before we actually list the results and analyze them, there is at least one major caveat that needs to be admitted. Unlike the previous section, where we looked only at ReFresh students, any comparison of ReFresh and non-ReFresh students is likely subject to serious issues of selection bias. Since ReFresh involves student selfselection - they must choose to take ReFresh - it is likely that the composition of the ReFresh population is not the same as the non-ReFresh population. But precisely how the two populations differ is a lot harder to tease out, based solely on these questions:

- Are ReFreshers more serious about academics, and choose the program for this reason?

- Or are they more insecure or simply weaker academically, and recognize a need to mitigate those problem-areas?

- Are ReFreshers disproportionately the kind of students who suffer from personal and psychological obstacles to their success?

- Or is the proportion of all of these various types of students within ReFresh approximately equal to the proportion that we would find in the non-ReFresh population?

Logic suggests that any self-selection process creates bias of some kind in the two population sets, and our discussion of the data below suggests that the second type of bias is the most likely. Therefore the validity of any comparison of the two groups must be seriously examined and contextualized in order to be made useful.

There is at least one important difference that we are aware of: ReFresh includes students who posted grades below $45 \%$ in their first attempt, and those students are not allowed to repeat 1 st year without ReFresh. As a result, they will be absent from the non-ReFresh population. Again, though, it's not entirely clear whether that necessarily weakens the ReFresh group in their second attempt at the Fall term. As shown in the previous section, there is little predictive value in first attempt grades when examining second attempts at the Fall term. However, self-selection is again a possible bias, since $<45 \%$ students are also much more likely to forego a second-attempt in Engineering, often transferring to another department upon completion of ReFresh. So, it may be that those $<45 \%$ students who repeat are relatively strong, at least among that particular peer-group, and do not skew the data. Again, these are questions that can't be adequately answered by the numbers, alone.

Table 2: Performance of all $<50 \%$ students since 2008

\begin{tabular}{|l|l|l|l|c|}
\hline & RF & Non RF & $\mathbf{R F}>\mathbf{4 5}$ & $\begin{array}{l}\text { Non RF } \\
>\mathbf{4 5}\end{array}$ \\
\hline $\begin{array}{l}\text { Repeated } \\
\text { Fall Avg }\end{array}$ & 62.6 & 63.1 & 67.8 & 62.7 \\
\hline $\begin{array}{l}\text { Winter } \\
\text { Avg }\end{array}$ & 60.9 & 64.6 & 62.3 & 61.7 \\
\hline $\begin{array}{l}\text { \% } \\
\text { students } \\
\text { to Repeat } \\
\text { Fall }\end{array}$ & $64 \%$ & $35 \%$ & $77 \%$ & $47 \%$ \\
\hline $\begin{array}{l}\text { \% to } \\
\text { proceed } \\
\text { to Winter }\end{array}$ & $47 \%$ & $27 \%$ & $62 \%$ & $32 \%$ \\
\hline $\begin{array}{l}\text { \% to } \\
\text { finish } \\
\begin{array}{l}\text { Winter } \\
>50\end{array}\end{array}$ & $45 \%$ & $27 \%$ & $62 \%$ & $32 \%$ \\
\hline
\end{tabular}

Table 2 - Comparison of the relative performances of ReFresh (RF) and non-ReFresh (non-RF) students. The $>45$ columns refer to performance in first attempt at Fall semester

We'll address the biggest questions first: there is basically no difference between a ReFresh student and a non-ReFresh student when it comes to their $2^{\text {nd }}$ attempt at the Fall term. This does not necessarily mean that ReFresh has no effect on their academic performance, but it is obviously not great news.

As for the good news: if we examine only those students who did well as previously defined in Table 1 with $>80 \%$ in ReFresh and clearly learned something in ReFresh, the average is $69 \%$, which is considerably better than the non-ReFresh average. We are inclined to conclude, then, that those students who both make a genuine attempt in ReFresh and do well do, indeed, see some benefit from ReFresh, though it's impossible to determine how much of the gain is actually attributable to the program.

There's another interesting way to parse the data, which is to compare only those students who scored between 45 and $49 \%$. This group excludes retroactive withdrawals (WDR), and students who scored below $45 \%$ the first time, since all of the latter category are ReFresh students. As seen in Table 2, in this category the ReFresh students clearly outperform the non-ReFresh students (by about $5 \%$ ) in the $2^{\text {nd }}$ attempt at Fall, though the difference is negligible in the Winter.

Retention is the more obvious success of the program, at least for the first year. Though not included in the 
above-table, $24 \%$ of all students who score below $45 \%$ in their $1^{\text {st }}$ attempt at the Fall term return for a $2^{\text {nd }}$ attempt. This is entirely due to ReFresh, as most students who score $<45 \%$ and choose not to enrol in ReFresh don't enrol in any academic program during the winter semester and therefore can't provide evidence to bolster their application for re-admission to $\mathrm{U}$ of $\mathrm{T}$ Engineering. Students are told that passing ReFresh can give them a much better chance of re-admission if their first semester marks are $<45 \%$ so many that don't do the program are self-selecting out of Engineering at $\mathrm{U}$ of $\mathrm{T}$ entirely. Though it must be noted that some students who complete ReFresh immediately state they don't want to return to the Faculty, but do want to stay in school and improve their academics and personal growth.

On the whole, ReFreshers are roughly twice as likely to return to the faculty as are non-ReFreshers, and equally likely to succeed in their $2^{\text {nd }}$ attempt at the Fall and progress to the Winter, as well as equally likely to progress from the Winter to second-year (Table 2). Again, as a percentage, this is not an obvious success; however, in terms of volume, ReFresh is successful. However, this could be another form of bias at work - it is entirely likely that students who take ReFresh are simply more inclined to return. Nevertheless, and this is admittedly speculation, ReFresh likely plays a role in improving the odds, turning that inclination into something more substantial, whereas a student who does not take ReFresh is likely to consider other options.

In fact, we would argue that this data is of very limited usefulness without some sort of accompanying qualitative data - Why did the students choose to do ReFresh? Why did other students elect to not do it? - which would confirm whether and what kind of bias needs to be accounted for. These questions can be considered in further study of a qualitative nature.

\section{CONCLUSIONS}

Failing for the first time can be an extreme shock to first year students who were once accustomed to easy success. This shock, though, can be a valuable teaching moment for a first year student, as s/he may never have reflected upon what they really want to study and how hard they need to work to achieve their goals. The opportunity to fail with a safety net of the ReFresh program can give students time to reflect and grow in a nurturing academic setting where they can get one-on-one support and attention from their instructors and TA's staff members as they assess their situation and review their goals. As can be seen from the data, ReFresh has some positive correlations with performance when students return to repeat their first year, specifically, those who demonstrate their aptitude during the program. While further qualitative research could be performed to enhance the data provided, it is safe to say that ReFresh either benefits or does not take away from most students' chances for success and gives them an opportunity to reflect as they continue their studies.

\section{Acknowledgements}

Leslie Grife, Assistant Registrar, First Year must be acknowledged as Lesley Mak's co-creator of ReFresh. Credit must also be given to Prof Susan McCahan, then Chair of First Year (now Vice Dean Undergrad) and Barbara McCann, Faculty Registrar, for greenlighting and supporting the ReFresh Program. The efforts of all the graduate students who instructed and TA'd the courses as well as the seminar presenters were also invaluable.

\section{References}

[1] Vincent Tinto, "Research and Practice of Student Retention: What Next?” Journal of College Student Retention Theory and Practice, vol. 8, no. 1, pp. 1-19, 2007. 